\title{
Convergent dynamics in the protease enzymatic superfamily
}

\author{
Vincenzo Carnevale, Simone Raugei, Cristian Micheletti and Paolo Carloni \\ International School for Advanced Studies (SISSA) and INFM Democritos, Via Beirut 2-4, I-34014 Trieste, ITALY
}

(Dated: October 10, 2018)

\begin{abstract}
Proteases regulate various aspects of the life cycle in all organisms by cleaving specific peptide bonds. Their action is so central for biochemical processes that at least $2 \%$ of any known genome encodes for proteolytic enzymes. Here we show that selected proteases pairs, despite differences in oligomeric state, catalytic residues and fold, share a common structural organization of functionally relevant regions which are further shown to undergo similar concerted movements. The structural and dynamical similarities found pervasively across evolutionarily distant clans point to common mechanisms for peptide hydrolysis.
\end{abstract}

\section{INTRODUCTION}

Proteases (PR's hereafter) perform enzymatic cleavage of peptide bonds in an enormous variety of biological processes 1 including cell growth, cell death, blood clotting, immune defense and secretion. Viruses and bacteria use PR's for their life cycle and for infection of host cells, rendering proteases key targets for antiviral and anti-bacterial intervention. PR's enzymatic action is accomplished by a wide repertoire of possible residues, Ser, Asp, Cys, Glu and Thr or even metal ions, giving rise to six different classes of enzymes. The enzymatic reaction is believed to involve in all cases a nuclephilic attack on a specific amide carbon belonging to the substrate main chain. The nucleophilic agent can be (a) the OH or the SH group of the namesake residues in Ser-Thr and Cys proteases; (b) a water molecule activated by the presence of an aspartic dyad or of a glutamate for Asp and Glu proteases; (c) a Zn-bound water molecule or OH group in metalloproteases ${ }^{1,2.3}$.

The large variety of catalytic active sites is paralleled by significant sequence and structural diversity: The approximately 2,000 proteases of known structure can, in fact, be assigned to as many as thirteen distinct folds ${ }^{1}$. Several attempts have been made to identify common features across the various protease folds and clans. So far, the only trait apparently shared by PR's is the fact that the peptide substrate in the catalytic cleft takes an extended $\beta$-conformation 4 . Here, by employing a novel quantitative methodological framework we extend significantly previous investigations of PR relatedness. First, by using bioinformatics tools we show that a previously unnoticed and statistically-significant structural correspondence exists among a dozen distinct protease clans. Such relatedness was previously pointed out only among the two known folds of cytoplasmatic aspartic proteases, namely pepsins and retropepsins ${ }^{5}$. Remarkably, extensive molecular dynamics simulations ${ }^{6.7}$ revealed qualitativelysimilar large scale movements for these Asp PR folds. Prompted by this fact we next carry out a systematic investigation of common functional dynamics in all pairs of structurally-related PR's. This step is accomplished within a novel framework, based on coarse-grained elastic network models ${ }^{8,9,10,11,12,13}$, which is straightforwardly transferable to other enzymatic superfamilies. Through this effective quantitative strategy we unveil the unsuspected and pervasive similarity of large-scale dynamical fluctuations that accompany concerted rearrangements for many, albeit not all, pairs of PR folds. The extensive comparison 
of structural and dynamical features across the entire set of PR folds suggests that several PR's besides Asp proteases share common conformational fluctuations impacting on their biological function.

\section{METHODS}

Structural bioinformatics. A set of reference structures of PR's common folds ${ }^{4}$ were selected using criteria of minimal sequence and structural redundancy. To this purpose the set of 1,928 presently-determined PR's structures, comprising 13 major folds, was intersected with the PDBselect ${ }^{14}$ list of structurally-resolved proteins with sequence identity smaller than $25 \%$, i.e. below the twilight zone of structural similarity 15 . This lead to a set of 69 structures, covering all seven common folds. For a comprehensive coverage of PR structural diversity we subdivided the structures according to the CATH criteria for class, architecture and topology ${ }^{16}$. For all common folds, A-G, several structures shared the same CATH labelling. For each of these groups we retained the entry with most complete PDB structure and, whenever available, in complex with a ligand. For the uncommon folds (i.e. folds represented by one or very few non-redundant PDB structures) we used the same representatives

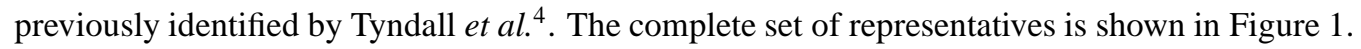

Common structural traits were next sought with the DALI algorithm ${ }^{17}$ in the 136 disctinct pairs of our representatives. DALI identifies blocks of residues having similar inter-residue distances. The consistency of the pairwise residue distances in two matching regions (based on the three-dimensional structure of the main chain with no sequence information) is measured by means of a knowledge-based score, $\sigma$. The optimal alignment returned by DALI is the one maximising the score, $\sigma_{o p t}$, and can comprise several distinct blocks. The order in which matching blocks appear in one protein is not necessarily the same in the partner one and the sequence directionality in two corresponding blocks may be reversed. These features endow DALI with considerable flexibility for identifying regions with common structural organization. The statistical relevance of the optimal DALI score $\sigma_{o p t}$ is quantified by the standard Z-score $=\frac{\sigma_{o p t}-\sigma_{a v e}}{\Delta \sigma_{a v e}}$, where $\sigma_{a v e}$ and $\Delta \sigma_{a v e}$ are, respectively, the average score and dispersion expected for structurally-unrelated proteins of length equal to the aligned ones. Assuming that probability distribution of $\sigma$ is approximately Gaussian, one has that alignments with Z-score greater than 2 probability ought to have a probability smaller than $2 \%$ to be generated by chance ${ }^{17}$. Finally, the oligomeric state of each active unit was fully taken into account in the structural alignments by merging all the polypeptide chains found in the biological unit deposited in the PDB and by running DALI on the "merged" chains. Accordingly, the resulting optimal alignments turned out to be, in general, different from that deposited in the DALI/DCCP database in which each chain is considered separately ${ }^{18}$.

Protein large scale motions. Prompted by Ref. 6 , in which a similarity of large-scale motions was established between the two known folds of cytoplasmatic Asp proteases, we aimed at establishing the consistency of the slow modes of each aligned pair of PR representatives $X$ and $Y$.

In several contexts these concerted rearrangements have been shown to be conditioned, and hence well-described, by the slowest modes of fluctuation around an enzyme's average structure ${ }^{19,20,21}$. Well-established procedure exist for calculating such modes in MD simulation contexts (i.e. by principal components analysis of the covariance matrix) ${ }^{22.23}$. The reliable identification 

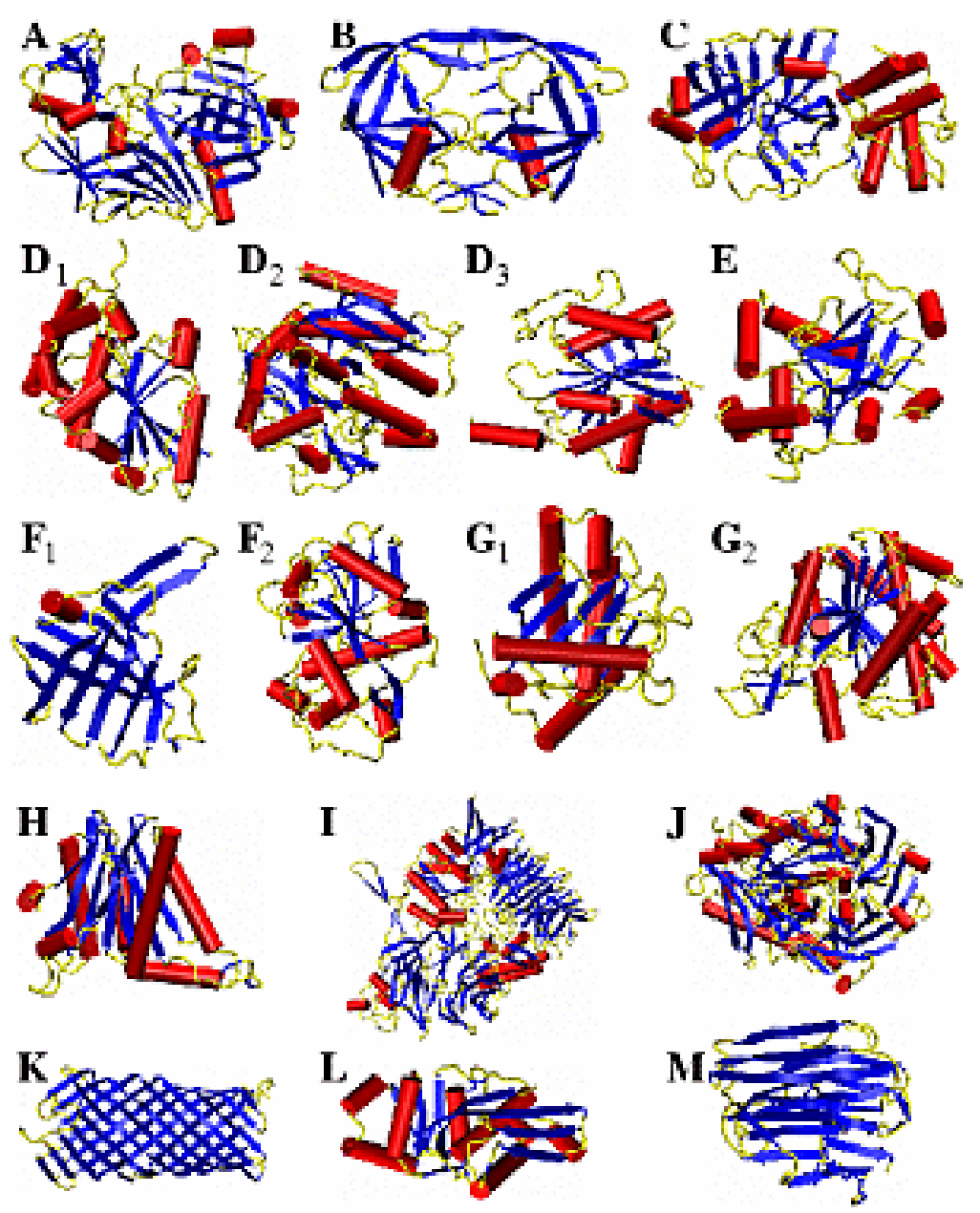

FIG. 1: Common (A-G) and uncommon PR folds (H-M). PDB codes ${ }^{44}$ and length of representatives are as follows: A 1er8 (330), B 1nh0 (198), C 1uk4A (302), D 1 lavp (204), D 1 ga6 (369), D 3 1ioi (208), E 1jq7A (210), F 1 1k3bA (119), F F $_{2}$ me4 (215), G lkuf $_{1}(201), \mathrm{G}_{2} 8 \mathrm{cpa}$ (307), H 1pmaA (221), I 1n6e (1023), J 1qfs (710), K 1i78A (297), L 1rr9A (182), M 1s2k (199).

of the essential spaces typically requires the monitoring of the system evolution over tens of nanoseconds $\stackrel{24}{ }$, entailing a very onerous computational expenditure for proteins of a few hundred amino acids. It is therefore apparent that such analysis cannot be carried out for each of the 17 PR representatives under consideration. We have hence resorted to a coarse-grained model, the $\beta$-Gaussian network mode $1 \underline{13}$, which provides a reliable (by comparison against atomistic simulations) description of concerted large-scale rearrangements in proteins with a negligible computational expenditure. In this approach, the concerted motions are calculated within the quasi-harmonic approximation of the free energy $\mathcal{F}$ around a protein's native state (assumed to coincide with the crystallographic structure). Thus, a displacement from the native state $\delta \vec{R}=\left\{\delta \vec{r}_{1}, \delta \vec{r}_{2}, \ldots, \delta \vec{r}_{N}\right\} \quad\left(\vec{r}_{i}\right.$ being the displacement of $\mathrm{C} \alpha$ atom $i$ ) is associated to the change in free energy $\Delta \mathcal{F} \approx \frac{1}{2} \delta \vec{R}^{\dagger} \mathbf{F} \delta \vec{R}$, where $\mathbf{F}$ is an "interaction" matrix constructed from the knowledge of contacting $C_{\alpha}$ and $C_{\beta}$ centroids in the native state $\frac{13}{3}$ and the $\dagger$ superscript indicates the transpose. The large scale motions of the system correspond to the eigenvectors of $\mathbf{F}$ having the smallest non-zero eigenvalues $\underline{13}$.

As we are interested only in the concerted motions of aligned regions of PR pairs, we subdivide the residues of each representative $X$ and $Y$ in two sets according to whether they take part to the DALI-aligned regions (set $A$, characterized by displacements 
$\delta \vec{R}_{A}$ ) or not (set $B$, characterized by $\delta \vec{R}_{B}$ ). Residues in set $A$ are ordered so that amino acids in structural correspondence appear in the same order for the two proteins. $\Delta \mathcal{F}$ then reads:

$$
\Delta \mathcal{F}=\frac{1}{2}\left(\delta \vec{R}_{A}^{\dagger} \delta \vec{R}_{B}^{\dagger}\right)\left(\begin{array}{cc}
\mathbf{F}_{A} & \mathbf{G} \\
\mathbf{G}^{\dagger} & \mathbf{F}_{B}
\end{array}\right)\left(\begin{array}{l}
\delta \vec{R}_{A} \\
\delta \vec{R}_{B}
\end{array}\right)
$$

where $\mathbf{F}_{A}\left[\mathbf{F}_{B}\right]$ is the interaction matrix within set $a \mathrm{~A}[B]$ and $\mathbf{G}$ contains the pairwise couplings across the two sets. The probability of occurrence of displacements $\delta \vec{R}_{A}$ and $\delta \vec{R}_{B}$ in thermal equilibrium is given by the Boltzmann distribution. Neglecting the normalization factor, it reads:

$$
P\left(\delta \vec{R}_{A}, \delta \vec{R}_{B}\right)=\exp \left(-\frac{\Delta \mathcal{F}}{k T}\right)=\exp \left(-\frac{\delta \vec{R}_{A}^{\dagger} \mathbf{F}_{A} \delta \vec{R}_{A}+\delta \vec{R}_{B}^{\dagger} \mathbf{F}_{B} \delta \vec{R}_{B}+2 \delta \vec{R}_{A}^{\dagger} \mathbf{G} \delta \vec{R}_{B}}{2 k T}\right)
$$

Since we focus only on the free energy change associated with residues in set $A$, we calculate the probability distribution for set $A$ integrated over all displacements in set $B$. The integration can be evaluated analytically and yields 25

$$
\tilde{P}\left(\delta \vec{R}_{A}\right)=\exp \left(-\frac{\Delta \mathcal{F}_{A}}{k T}\right)=\int d \delta \vec{R}_{B} P\left(\delta \vec{R}_{A}, \delta \vec{R}_{B}\right) \propto \exp \left[-\left(\frac{\delta \vec{R}_{A}^{\dagger}\left(\mathbf{F}_{A}-\mathbf{G ~ F ~ F}_{B}^{-1} \mathbf{G}^{\dagger}\right) \delta \vec{R}_{A}}{2 k T}\right)\right]
$$

hence:

$$
\Delta \mathcal{F}_{A}=\frac{1}{2} \delta \vec{R}_{A}^{\dagger}\left(\mathbf{F}_{A}-\mathbf{G ~ F}_{B}^{-1} \mathbf{G}^{\dagger}\right) \delta \vec{R}_{A}
$$

Thus, the eigenvectors associated with the smallest eigenvalues of $\left(\mathbf{F}_{A}-\mathbf{G ~ F}_{B}^{-1} \mathbf{G}^{\dagger}\right)$ represent the integrated slow modes of the matching regions; the term "integrated" is used to stress the fact that the modes depend also on the non-matching ones via the contributions $\mathbf{G}$ and $\mathbf{F}_{B}$. The eigenvectors of $\mathbf{F}_{A}$, instead, will be termed "bare" slow modes since they neglect the presence of the non-matching regions. The comparison of the integrated and bare essential dynamical spaces is used here to investigate the influence of the non-matching regions over the dynamics of the matching ones.

The eigenvectors of $\left(\mathbf{F}_{A}-\mathbf{G ~ F}_{B}^{-1} \mathbf{G}^{\dagger}\right)$, calculated separately for proteins $X$ and $Y$, can be directly compared component by component (we assume that $X$ and $Y$ are represented in the Cartesian coordinates set providing the optimal structural superposition of the DALI matching regions). To measure the agreement of the integrated dynamics of proteins $X$ and $Y$ we hence considered the root mean square inner product (RMSIP) of the top 10 slowest modes $\vec{v}_{1, . ., 10}^{X}$ and $\vec{v}_{1, ., 10}^{Y}$ of $\left(\mathbf{F}_{A}-\mathbf{G ~ F}_{B}^{-1} \mathbf{G}^{\dagger}\right)^{26}$,

$$
\operatorname{RMSIP}(\operatorname{set} A)=\sqrt{\sum_{i, j=1, . ., 10}\left|\vec{v}_{i}^{X} \cdot \vec{v}_{j}^{Y}\right|^{2} / 10}
$$

If a comparison is sought for the "bare" dynamics, the eigenvectors of $\mathbf{F}_{A}$ are used in place of those of $\left(\mathbf{F}_{A}-\mathbf{G ~ F}_{B}^{-1} \mathbf{G}^{\dagger}\right)$. The value taken on by the RMSIP, ranging from 0 (complete absence of correlation) to 1 (exact coincidence of the slow modes), is compared with a control RMSIP distribution to assess its statistical significance. The term of comparison is given by the 
distribution of RMSIP values resulting by randomly choosing the residues in set $A$, that is for arbitrary choices of the blocks of corresponding residues in structures $X$ and $Y$. Accordingly, we stochastically generated 100 "decoy" sets of matching residues in $X$ and $Y$ involving the same number of amino acids as the optimal DALI alignment of $X$ and $Y$. Also the typical size of DALI matching blocks (10-15 residues) is respected in the control alignments. For each stochastic alignment we carried out numerically the dynamical integration described above and hence obtained the corresponding RMSIP value from equation (4). By processing the results of the 100 decoy alignments we calculated the average value and dispersion of the control RMSIP distribution, $\langle$ RMSIP $\rangle$ and $\triangle \mathrm{RMSIP}$. These quantities were used to define the dynamical Z-score: $\left(\mathrm{RMSIP}_{D A L I}-\right.$ $\langle$ RMSIP $\rangle) / \Delta$ RMSIP. In analogy to the structural Z-score, it provides a measure of how unlikely it is that the RMSIP of the DALI matching regions could have arisen by chance.

The viability of this procedure for comparing the large-scale movements of the matching residues in two proteins was tested within the context of atomistic MD simulations in aqueous solution. In particular, the analysis was carried out on two trajectories of 10 and 20 ns previously obtained by us for HIV-1 PR and BACE, respectively ${ }^{6.7}$. Since dynamical trajectories are available, it is not necessary to resort to the $\beta$-Gaussian model for calculating the integrated essential dynamical spaces of set $A$. The latter are, in fact, calculated from the covariance matrix constructed for the matching regions alone (i.e. removing the roto-translation of the latter $)^{27}$. The standard definition of covariance matrix is employed, i.e. the generic matrix element reads

$$
C_{i j, \alpha \beta}=\left\langle\delta \vec{r}_{i}^{\alpha} \cdot \delta \vec{r}_{j}^{\beta}\right\rangle_{t}
$$

where \langle\rangle$_{t}$ denotes the time average of the displacements (at equal times) of residues $i$ and $j$ corresponding to the Cartesian components $\alpha$ and $\beta$. Within our quadratic approximation for the free energy, the principal components of the covariance matrices exactly correspond to the slow modes. For this reason we shall measure the dynamical accord in the atomistic simulations context by considering both the RMSIP calculated over the principal spaces of the matrices $C_{X}$, and $C_{Y}$ as well as from the similarity of corresponding entries in the two matrices. The dynamical RMSIP of the DALI matching regions obtained from this approach was found to be equal to 0.65 , consistently with the high statistical significance of corresponding entries of the two normalised reduced covariance matrices ${ }^{13}, \tilde{C}$, (definition: $\tilde{C}_{i j}=\sum_{\alpha} C_{i j, \alpha \alpha} / \sqrt{\sum_{\alpha, \beta} C_{i i, \alpha \alpha} \sum_{\alpha} C_{j j, \beta \beta}}$ ) as visible in Figure 2 


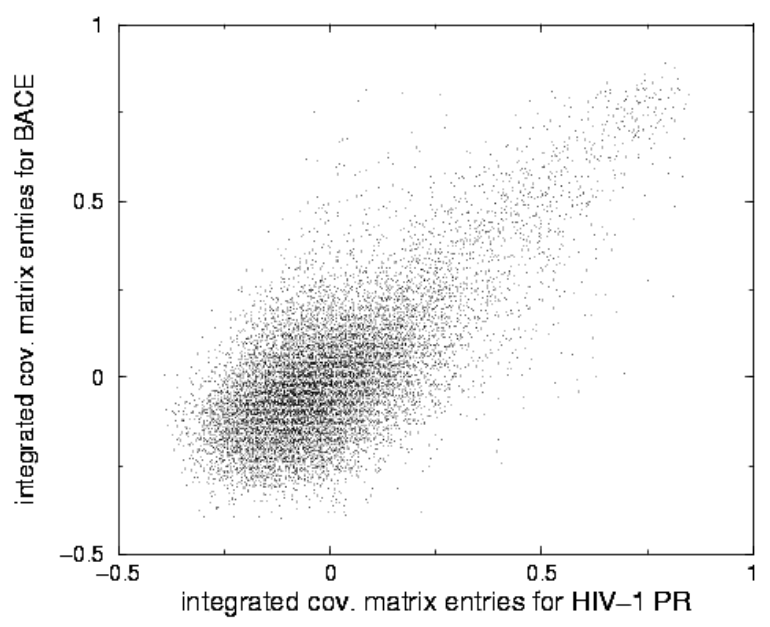

FIG. 2: Scatter plot of corresponding matrix elements of the integrated reduced normalised covariance matrices for BACE and HIV-1 PR obtained from MD simulations in explicit solvent. The linear correlation coefficient of the 14,000 distinct entries is 0.77 . The non-parametric Kendall correlation coefficient is instead, $\tau=0.41$ corresponding to an extremely large statistical significance $(z \sim 70)$.

\section{RESULTS AND DISCUSSION}

\section{Structural alignment across PR's.}

(i) Identification of representatives. The set of 1,928 presently-known proteases was initially reduced to a collection of 69 entries with minimal mutual sequence identity. The resulting structures, which covered the whole range of common folds, were then subdivided according to the CATH criteria for class, architecture and topology $y^{16}$. For each CATH entry, we then selected the most complete structure and, whenever available, one in complex with a peptide mimic substrate. For the six uncommon folds we retained, instead, the representatives previously identified by Tyndall et al $\stackrel{4}{\text {. }}$. The 17 representatives are shown in Figure 1 Besides the major structural differences across folds it is interesting to notice that folds D, F and G possess a fair degree of internal structural heterogeneity at the "topology" level of the CATH classification scheme ${ }^{16}$ and that only $\mathrm{D}_{3}$ and $\mathrm{G}_{2}$ are exopeptidases. Except for representatives $F_{1}$ and $F_{2}$ and those of pepsins and retropepsins (folds $A$ and $B$ ), all other reference structures belong to distinct clans according to the MEROPS classification $\stackrel{1}{*}$. Since this is indicative of a different evolutionary origin, any common property found persistently in members of Figure 1 arguably reflects a convergent evolutionary pressure.

(ii) Alignment of pairs of representatives. Because of the major structural differences across PR's, global structural matches of the representatives were not attempted. Rather, we looked for partial structural alignments among all the representative pairs (136) using the DALI algorithm ${ }^{17}$. DALI identifies corresponding blocks of residues having similar inter-residue distances (with either direct or reversed sequence directionality), and provides a score function (Z-score), conveying the statistical significance of the alignment. Values of Z-score $\sim 2$ or larger, corresponds to alignments expected to have a probability of less than $2 \%$ to be generated by chance. The top 20 pairs having Z-score $>\sim 2$ are shown in Table

Such alignments are typically constituted by several disconnected matching blocks with the same directionality. This is ilustrated in Figure 3 which portrays statistically-relevant alignments against representatives $\mathrm{D}_{3}$ (PDB entry 1IOI) and B (1NH0). 


\begin{tabular}{|c|c|c|c|c|c|c|c|}
\hline \begin{tabular}{|c|} 
Fold from \\
protein 1 \\
\end{tabular} & \begin{tabular}{|c|} 
Fold from \\
protein 2 \\
\end{tabular} & Length & $\begin{array}{r}\text { Seq. Id. } \\
(\%)\end{array}$ & $\begin{array}{c}\text { RMSD } \\
(\AA) \\
\end{array}$ & \begin{tabular}{|r||} 
DALI \\
Z-score
\end{tabular} & \begin{tabular}{|r|} 
Dynamical \\
RMSIP \\
\end{tabular} & $\begin{array}{r}\text { Dynamical } \\
\text { Z-score }\end{array}$ \\
\hline A $\quad$ (Asp) & B $\quad$ (Asp) & 168 & 14 & 3.7 & $10.4 \|$ & 0.71 & 36.86 \\
\hline (Ser) & (Ser) & 257 & 11 & 5.6 & 8.9 & 0.70 & 11.56 \\
\hline $\mathbf{D}_{3}$ (Cys) & $\mathbf{G}_{2}$ (Met) & 150 & 7 & 3.3 & $8.8 \|$ & 0.74 & 10.56 \\
\hline $\mathrm{J} \quad($ Ser $)$ & $\mathrm{G}_{2}$ (Met) & 151 & 6 & 4.0 & $5.2 \|$ & 0.70 & 11.85 \\
\hline $\mathrm{D}_{3}$ (Cys) & $\mathrm{J} \quad$ (Ser) & 101 & 10 & 3.0 & 4.2 & 0.70 & 10.18 \\
\hline $\mathrm{K} \quad(\mathrm{Asp})$ & $F_{1} \quad(\mathrm{Cys})$ & 84 & 10 & 3.9 & 4.0 & 0.65 & 15.71 \\
\hline $\mathrm{D}_{2} \quad$ (Ser) & I $\quad$ (Ser) & 130 & 8 & 4.8 & $3.8 \|$ & 0.58 & 5.57 \\
\hline $\mathbf{D}_{2}$ (Ser) & $\mathbf{D}_{3}$ (Cys) & 95 & 6 & 4.6 & $3.3 \|$ & 0.69 & 8.70 \\
\hline $\mathbf{D}_{2}$ (Ser) & $\mathbf{G}_{1}$ (Met) & 125 & 6 & 4.5 & 3.2 & 0.71 & 12.07 \\
\hline $\mathrm{D}_{2}$ (Ser) & $\mathrm{J} \quad$ (Ser) & 134 & 7 & 3.9 & $3.1 \|$ & 0.72 & 9.33 \\
\hline $\mathrm{D}_{3}$ (Cys) & I $\quad$ (Ser) & 94 & 10 & 3.7 & 2.9 & 0.64 & 6.84 \\
\hline $\mathbf{D}_{3} \quad$ (Ser) & $\mathbf{G}_{1}$ (Met) & 89 & 8 & 3.4 & $2.7 \|$ & 0.67 & 7.34 \\
\hline $\mathbf{G}_{1}$ (Met) & $\mathbf{G}_{2}$ (Met) & 104 & 8 & 4.0 & 2.6 & 0.65 & 8.84 \\
\hline $\mathrm{G}_{1}$ (Met) & $\mathrm{L} \quad$ (Ser) & 73 & 1 & 3.0 & $2.3 \|$ & 0.70 & 9.37 \\
\hline $\mathrm{D}_{2}$ (Ser) & L $\quad$ (Ser) & 103 & 10 & 4.9 & 2.3 & 0.69 & 12.93 \\
\hline $\mathbf{D}_{2} \quad$ (Ser) & $\mathbf{G}_{2}$ (Met) & 138 & 9 & 4.5 & 2.1 & 0.65 & 6.55 \\
\hline I $\quad$ (Ser) & L $\quad$ (Ser) & 58 & 9 & 3.7 & 2.0 & 0.77 & 9.34 \\
\hline $\mathrm{D}_{3}$ (Cys) & L $\quad$ (Ser) & 65 & 11 & 3.8 & 1.9 & 0.73 & 8.62 \\
\hline $\mid F_{2} \quad($ Cys $)$ & H $\quad$ (Ser) & 54 & 7 & 3.2 & $1.8 \|$ & 0.68 & 7.15 \\
\hline B $\quad$ (Asp) & C $\quad$ (Ser) & 73 & 7 & 4.8 & $1.8 \|$ & 0.69 & 9.87 \\
\hline
\end{tabular}

TABLE I: Top 20 structural alignments of pairs of representative proteases ranked according to the statistical significance (DALI Z-score). The fold (see Figure 1 and chemical class of the pairs are provided in the first two columns. The total number of aligned residues is given in column 3 along with the sequence identity (Seq. Id.) and RMSD over the matching regions. Pairs of common folds are highlighted in boldface. The dynamical accord of the latter and the associated statistical significance are provided in the last two columns.

The former was chosen owing to the large number of significant alignments to which it takes part, while the latter provides

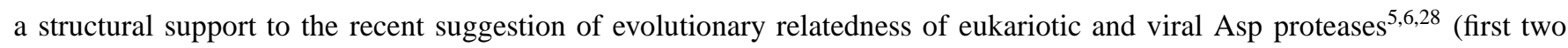
structures in Figure 1 .

As visible in Figure 4 the partial structural alignments (that we stress are oblivious to sequence specificity) typically present a superposition of the PR catalytic residues. Even more notable is the active site correspondence found in PR's with different catalytic residues (see Figure $4 \mathrm{~b}$ and c). The fact that alignments build around the active site implies a good consistency of the regions alignable against different PR's. This can be readily perceived in the pile-up diagrams of Figure 3

In contrast, the alignability of PR's with generic proteins is much poorer. This was established by considering the publiclyavailable DCCP listing of DALI alignments among several thousands of protein representatives 14.17 . We considered all alignments spanning more than 70 residues and involving at least one known protease. More than 80,000 such alignments were found which were ranked in terms of DALI Z-score. Within the 40,000 top alignments (Z-score greater than 6) we found that $70 \%$ of the matches involved protease pairs, not necessarily of the same class. In other words whenever a protease admits a structural alignment with high statistical significance, the partner protein is likely to be another protease. Since no sequence information is used by DALI, the high selectivity of matches involving PR's hints to a functional-basis for the observed structural correspondence. 
(a)
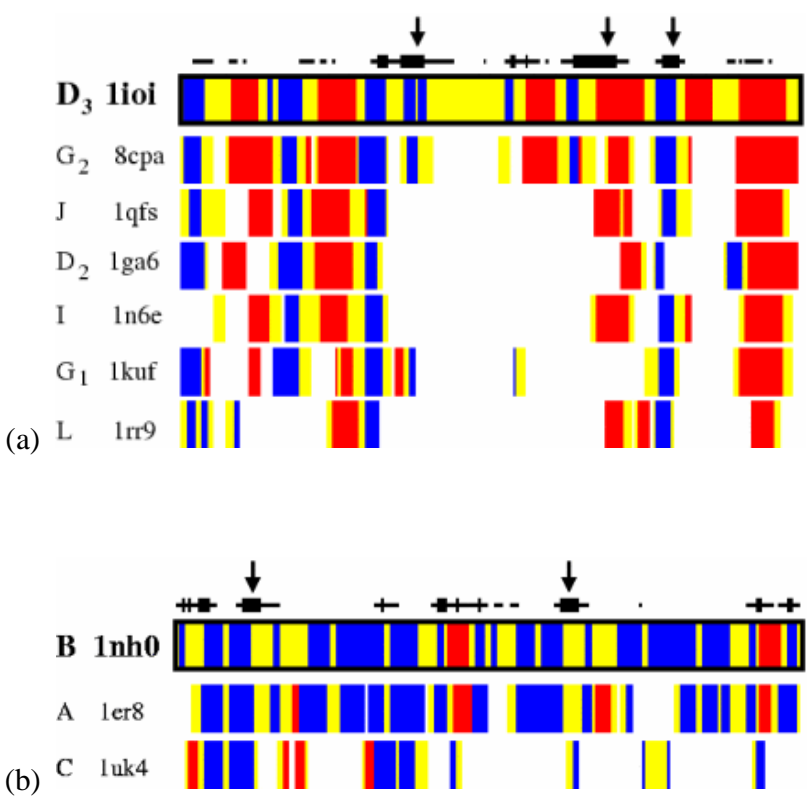

FIG. 3: Pile-up of alignments involving (a) fold $\mathrm{D}_{3}$ and (b) fold $\mathrm{B}$. The boxed panel is the linear representation of the secondary structure content of the reference protein (red: helix, blue: extended, yellow: loop/turn). Above the box: arrows indicate the location of the catalytic residues, thick [thin] segments indicate amino acids within 7 [10] $\AA$ of the catalytic sites. For each aligned protein we show, below the box, the location of the matching residues and the corresponding secondary content.

\section{Large-scale dynamics across PR's.}

The extent and significance of the structural matches found here, spanning members of distinct protease clans $\stackrel{1}{1}$, is suggestive of a biological selection criterion transcending the chemical determinants. An appealing possibility is the existence of an underlying unifying principle related to the necessity of proteolytic catalysis to rely on well-defined concerted functional movements.

Whilst the presence of concerted motions in enzymes is well-established $6,7,12,13,29,30,31,32,33,34,35,36,37,38,39$, the relevance of such movements for catalysis is a subject of vivid debate. Several groups $6,7,12,13,29,30,31,32,33,34,35,36,37,38$ have argued that these movements could be a result of specific protein architectures aimed at preserving the rigidity of the active site region. In the specific case of Asp proteases, different lines of research suggest that conformational fluctuations may play a role for the function ${ }^{6.7 .13 .34}$ and involve conserved structural features across the family ${ }^{5.6 .28}$.

Prompted by these suggestions, we extended the investigation of common large-scale dynamics to all pairs of PR representatives. To calculate the slowest modes (essential dynamical spaces ${ }^{26}$ ) of each representative we have used a relatively accurate and computationally affordable coarse-grained approach, the $\beta$-Gaussian network model $\stackrel{13}{ }$. This method was employed in a novel context (see Methods) which allows to describe the protein large-scale movements in the frame of reference of the matching regions. Since the dynamical influence of the non-matching ones is, nevertheless, taken into account we shall term the approach "integrated" to distinguish it from the "bare" description where the non-matching residues are entirely omitted.

Two indexes were used to identify the degree of correlation between the slow motions of two representative pairs for both the integrated and bare case. The first one is the so-called RMSIP, which provide a quantitative estimate of the consistency of the 10 slowest modes of the proteins (RMSIP $=0$ [1] corresponds to no [full] correlation). The second one is the dynamical Z-score 


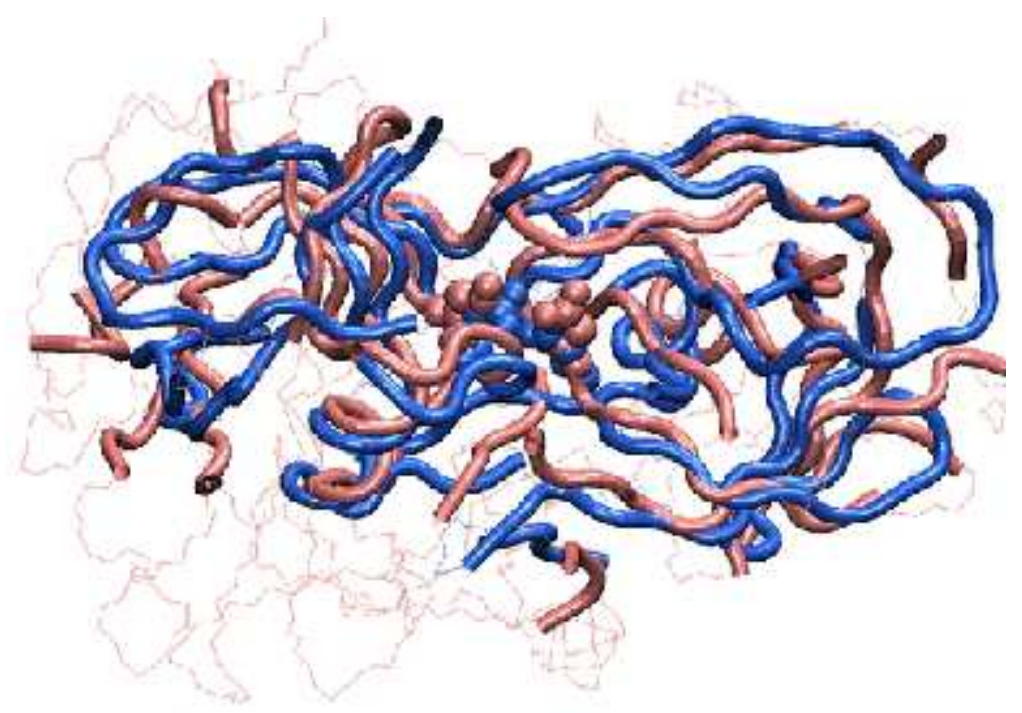

(a)

(b)

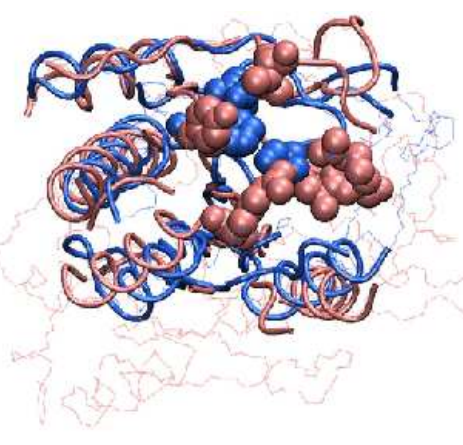

(c)

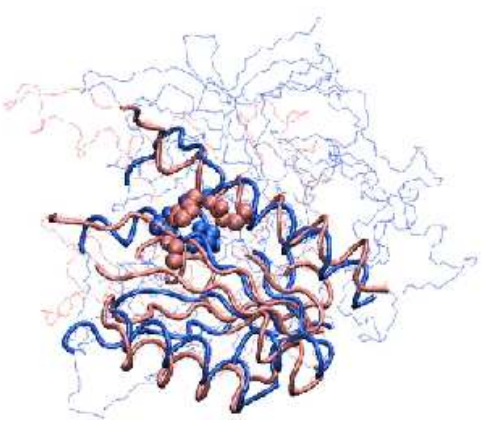

FIG. 4: Top structural alignments (according to Z-score) of common protease folds, see Table \ (a) endothiapepsin (ASP) and HIV-1 retropepsin (ASP) - folds A and B; (b) pyroglutamyl-peptidase I (Cys) and carboxypeptidase A1 (Zn)- folds D3 and G2; (c) pyroglutamylpeptidase I (Cys) and sedolisin (Ser) - folds D3 and D2. Catalytic residues are drawn as spheres. The thick backbone highlights the overlapping region.

which, in analogy with the structural one, measures the statistical significance of the observed accord (by comparison against randomly-generated "DALI-like" alignments).

The dynamical accord reported in Table \turns out not to capture a mere consistency of overall mobility, but reflects the close correspondence of the directionality of the slow modes at a residue-wise level. The inspection of the principal directions of the large-scale movements (see Figure 5) indicates prominent rearrangements of active site surroundings (i.e. flaps, cleavage, and recognition sites) resulting in a distortion of the crevice accommodating the substrate. This is suggestive of a common dynamical selection operated by the necessity to recognise/process peptides in well-defined geometrical arrangements (such as the $\beta$-extended one ubiquitously observed in bound PR substrate analogs $\left.{ }^{4}\right)$. Consistently, the integrated dynamical movements found here for Asp PR's appeared to be directly related to functional dynamics. In fact, the difference vector describing the structural distortion between inactive and reactive conformations of HIV-1 PR ${ }^{7.40}$ is mostly concentrated (91\% of the norm) on the regions that match with BACE. Furthermore, the top 10 slow modes of the matching regions are able to account for $71 \%$ of the norm of the difference vector over the same set of residues. 


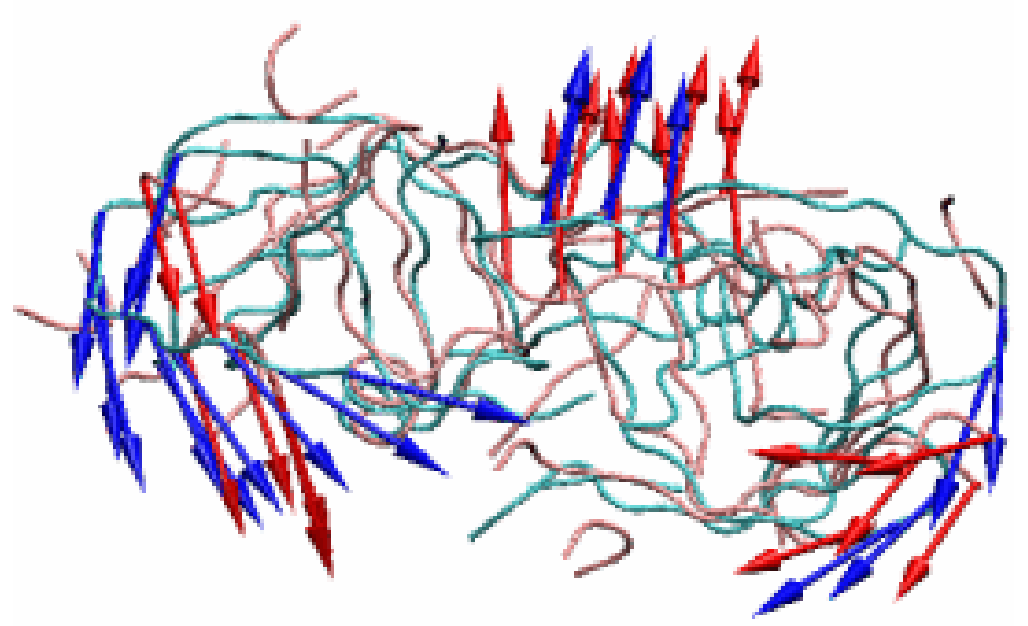

(a)

(b)

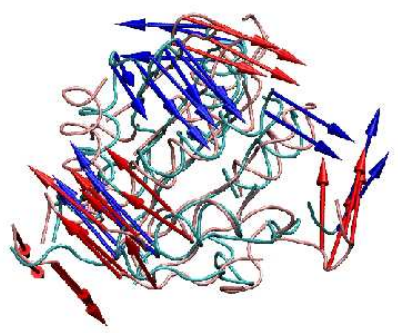

(c)

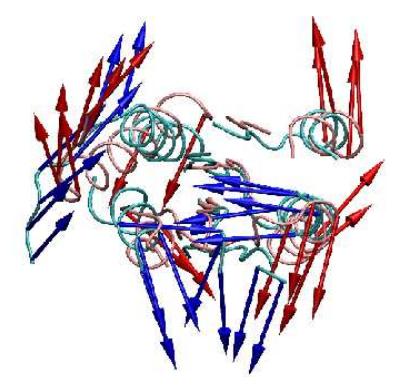

FIG. 5: Dynamical overlap for the same PR pairs of Figure 4 (a) endothiapepsin (ASP) and HIV-1 retropepsin (ASP) - folds A and B; (b) pyroglutamyl-peptidase I (Cys) and carboxypeptidase A1 (Zn)- folds D3 and G2; (c) pyroglutamyl-peptidase I (Cys) and sedolisin (Ser) folds D3 and D2. Red/pink and blue/cyan colors denote the dynamical and structural features of the aligned pairs. The top three essential dynamical spaces of the matching regions in the two proteins were considered. The directions of the 20 largest displacements of the best overlapping pair of modes are shown as arrows of equal length.

Several conclusions can be drawn. First, and most importantly, the RMSIP values for pairs showing statistically-significant alignment are clustered around 0.7 , which reflects an excellent degree of correlation ${ }^{26}$. In fact, this value exceeds by several times the one expected for random "DALI-like" alignments. Second, a highly significant structural alignment, e.g. Z-score $>4$, implies a strong integrated dynamical correspondence, dynamical Z-score $>10$, see Figure 6 This is indicative of a correlation between similar protein movements and structural similarity. However, no precise common trend exists between the structural and dynamical Z-scores, as visible in Figure 6 This may reflect the fact that the dynamical fluctuations play a different role in different members of the PR family. Third, the non-matching regions are not dynamically "neutral", but are co-opted for establishing the dynamical correspondence of the matching ones. In fact, when the non-matching regions are entirely omitted from the coarse-grained dynamical analysis (see Methods), the corresponding dynamical accord decreases dramatically ("bare" case of Figure 6 .

Finally, all these conclusions are robust against the use of other common measures of dynamical consistency and statistical relevance (e.g. linear or Kendall's correlation of covariance matrices) or if free enzymes, i.e. with no bound substrate, are used. 


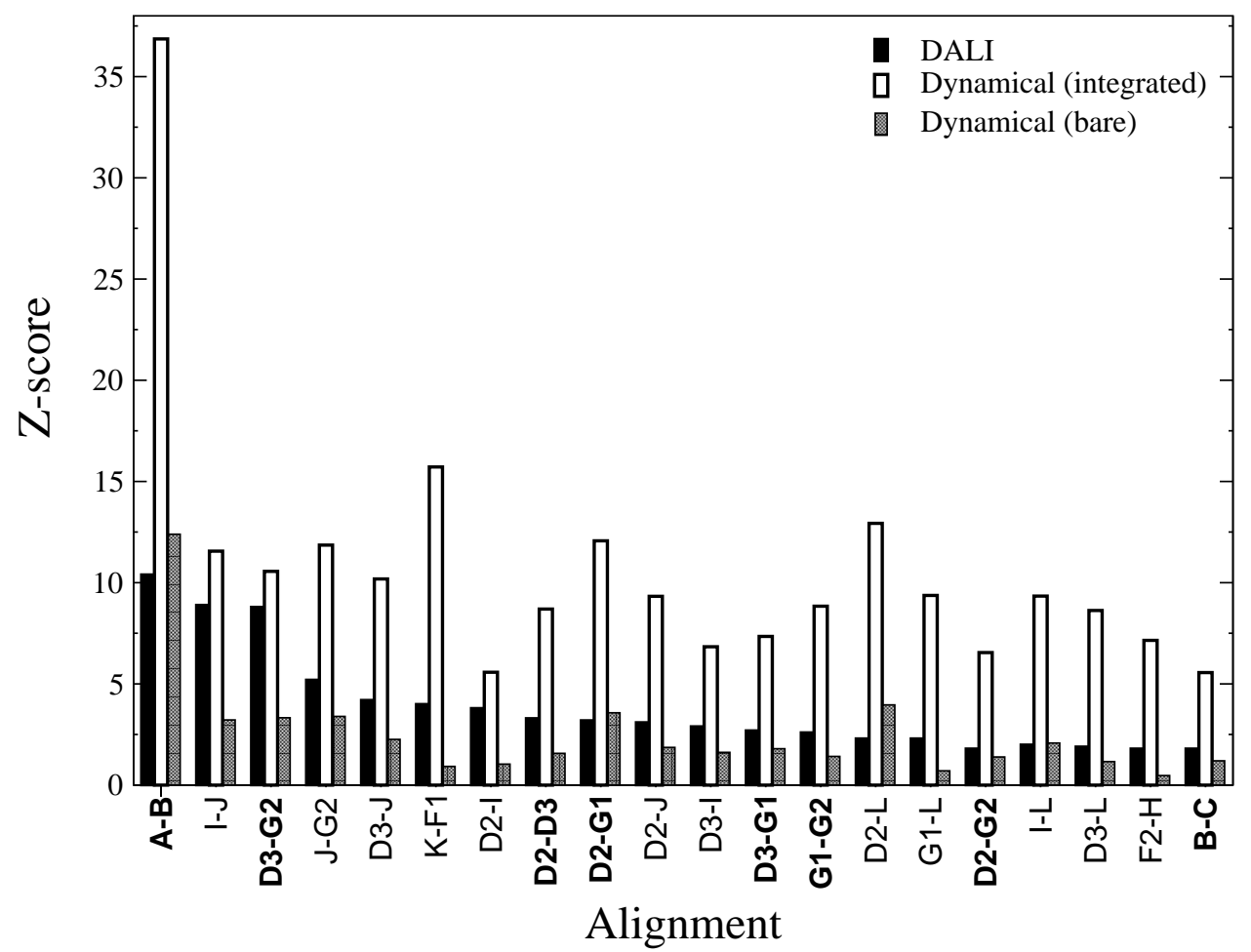

FIG. 6: Trend of the Z-scores for structural (DALI) and dynamical alignments for the $20 \mathrm{PR}$ pairs of Table The pairs are ranked according to the DALI Z-score. Pairs of common folds are highlighted in boldface. The dynamical Z-scores have been calculated using both the integrated approach and the bare one. The former accounts correctly for the dynamical influence of the non-matching regions, while the latter neglects it entirely (see Methods).

\section{Concluding remarks}

Large-scale motions, which certainly occur in enzymes $6.7 .12 .13,29.30 .31 .32 .33 .34 .35 .36 .37 .38$, have been increasingly suggested to play a role for enzymatic function for Asp PR's ${ }^{6,7,13,28,34}$ whilst it has not emerged for other major PR classes, notably Ser PR's. In the latter case, it has been strongly suggested by many groups that electrostatics is crucial for the enzyme ${ }^{41.42}$. It is therefore interesting to consider, the structural/dynamical alignments of common PR folds (highlighted in Table $\$ and Figure 6 in the light of these previous observations.

First, we notice that the Asp PR's (folds A and B) present the highest similarity of structural and dynamical features, providing further support to the functional relevance of conformational fluctuations for these enzymes. Second, any other fold exhibiting statistically-significant, yet far smaller, structural and dynamical scores involve a subfamily of Cys and Ser PR's, namely caspaselike and subtilisin-like, along with metallo-proteases. As conformational fluctuations are expected not to be determinant for Ser PR functionality ${ }^{41.42}$, it is tempting to conclude that these concerted motions might not play a critical role for enzymatic catalysis in this subfamily. The question of why such high degree of structural and dynamical similarity exists in this subfamily emerges spontaneously. An appealing, yet highly speculative answer, is the fact that the common features have been selected to maintain the active site relatively rigid and therefore efficient for Ser PR catalysis or to bind and recognize the substrate ${ }^{43}$. The lowest ranking alignment involving Asp PR's in Table \and Figure @ is between retropepsins (fold B) and the trypsin-like Ser PR 
representative (fold C) which, could not be aligned with any other fold.

In summary, across selected PR's with different folds and catalytic chemistry we observed a strong consistency of the essential dynamics around the active site. The intimate connection between the functional dynamics and enzymatic structure ${ }^{12}$ reverberates in strikingly-similar spatial organization of the regions surrounding the active site. This suggestes that evolutionary pressure may have resulted in a conservation across the family not only of the structural features but also of the dynamical ones. Considerable structural diversity is observed outside this region. Yet, this variability is not arbitrary but is co-opted to produce consistent large-scale dynamics of the functional region. In some specific cases, and when the dynamical and structural conservation has a high statistical significance, these results strongly suggest that the essential dynamical spaces have an important role for enzymatic catalysis.

Acknowledgment. This work was supported by INFM - Democritos. We are indebted to Martino Bolognesi and Arthur Lesk for discussions and comments on the manuscript.

1 A. J. Barrett, N. D. Rawlings, J. F. Woessner, and eds, Handbook of Proteolytic Enzymes; Elsevier, Amsterdam, second ed., 2004.

${ }^{2}$ L. Stryer, Biochemistry; Freeman W.H., New York, 4th ed., 1995.

3 L. Vandeputte-Rutten and P. Gros, Curr. Op. Struc. Biol., 2002, 12, 704-708.

4 J. D. Tyndall, T. Nall, and D. P. Fairlie, Chem. Rev., 2005, 105, 973-999.

5 T. L. Blundell and N. Srinivasan, Proc. Natl. Acad. Sci. USA, 1996, 93, 14243-14248.

${ }^{6}$ M. Cascella, C. Micheletti, U. Rothlisberger, and P. Carloni, J. Am. Chem. Soc., 2005, 127, 3734-3742.

7 S. Piana, P. Carloni, and M. Parrinello, J. Mol. Biol., 2002, 319, 567-583.

${ }^{8}$ M. M. Tirion, Phys. Rev. Lett., 1996, 77, 1905-1908.

9 A. R. Atilgan, S. R. Durell, R. L. Jernigan, M. C. Demirel, O. Keskin, and I. Bahar, Biophys. J., 2001, 80, 505-515.

10 T. Horiuchi and N. Go, Proteins, 1991, 10, 106-116.

11 I. Bahar, A. R. Atilgan, and B. Erman, Fold. \& Des., 1997, 2, 173-181.

12 O. Keskin, R. L. Jernigan, and I. Bahar, Biophys. J., 2000, 78(4), 2093-2106.

13 C. Micheletti, P. Carloni, and A. Maritan, Proteins, 2004, 55, 635-645.

${ }^{14}$ U. Hobohm and C. Sander, Prot. Sci., 1992, 2, 522.

15 A. M. Lesk, Introduction to Protein Science: Architecture, Function and Genomics; Oxford University Press, UK, 2004.

${ }^{16}$ F. Pearl, A. Todd, I. Sillitoe, M. Dibley, O. Redfern, T. Lewis, C. Bennett, R. Marsden, A. Grant, D. Lee, A. Akpor, M. Maibaum, A. Harrison, T. Dallman, G. Reeves, I. Diboun, S. Addou, S. Lise, C. Johnston, A. Sillero, J. Thornton, and C. Orengo, Nucleic Acids Research, 2005, 33, D247-D251.

${ }^{17}$ L. Holm and C. Sander, Science, 1996, 273, 595-603.

${ }^{18}$ L. Holm and C. Sander, Nucl. Acid Res., 1997, 25, 231-234.

19 J. A. McCammon, B. R. Gelin, M. Karplus, and P. G. Wolynes, Nature, 1976, 262, 325-326.

${ }^{20}$ V. Alexandrov, U. Lehnert, N. Echols, D. Milburn, D. Engelman, and M. Gerstein, Protein Science, 2005, 14, $633-643$.

${ }^{21}$ M. Delarue and Y. H. Sanejouand, J. Mol. Biol., 2002, 320(5), 1011-1024.

22 A.E. Garcia, Phys. Rev. Lett., 1992, 68, 2696-2699.

23 A. Amadei, A. B. M. Linssen, and H. J. C. Berendsen, Proteins, 1993, 17, 412-425.

24 B. Hess, Phys. Rev. E, 2002, 65, 031910.

${ }^{25}$ K. Hinsen, A. J. Petrescu, S. Dellerue, M. C. Bellisent-Funel, and G. Kneller, Chem. Phys., 2000, 261, $25-37$.

26 A. Amadei, M. A. Ceruso, and A. Di Nola, Proteins, 1999, 36, 419-424.

27 A. Pang, Y. Arinaminpathy, M. S. P. Sansom, and P. C. Biggin, Proteins: Structure, Function, and Bioinformatics, 2005, 61, 809-822.

${ }_{28}$ M. Neri, M. Cascella, and C. Micheletti, J. Phys. Cond. Mat., 2005, 17, 1581-1593.

29 T. H. Rod, J. L. Radkiewicz, and C. L. Brooks, Proc. Natl. Acad. Sci. USA, 2003, 100, 6980-6985.

30 R. M. Daniel, R. V. Dunn, J. L Finney, and J. C. Smith, Ann. Rev. Biophys. Biomol. Struct., 2003, 32, 69-92.

31 P. K. Agarwal, S. R. Billeter, P. T. Rajagopalan, S. J. Benkovic, and S. Hammes-Schiffer, Proc. Natl. Acad. Sci. USA, 2002, 99(5), 27942799.

32 A. Tousignant and J. N. Pelletier, Chem. Biol., 2004, 11, 1037-1042.

33 G. M. Suel, S. W. Lockless, M. A. Wall, and R. Ranganathan, Nat. Str. Biol., 2003, 10(1), 59-69. 
34 A. L. Perryman, J-H. Lin, and J. A. McCammon, Prot. Sci., 2004, 13, 1108-1123.

35 J. Luo and T. Bruice, Proc. Natl. Acad. Sci. USA, 2004, 101, 13152-13156.

${ }^{36}$ E. Z. Eisenmesser, D. A. Bosco, M. Akke, and D. Kern, Science, 2002, 295, 1520 - 1523.

37 I. Bahar, A.R. Atilgan, M.C. Demirel, and B. Erman, Phys. Rev. Lett., 1998, 80, 2733-2736.

38 C. Micheletti, G.L. Lattanzi, and A. Maritan, J. Mol. Biol., 2002, 321, 909-921.

39 M. H. M. Olsson, W. W. Parson, and A. Warshel, Chem. Rev., 2006, NA.

40 S. Piana, P. Carloni, and U. Rothlisberger, Prot. Sci., 2002, 11, 2393-2402.

41 A. Warshel, G. Naray-Szabo, F. Sussman, and J. K. Hwang, Biochemistry, 1989, 28, 3629-3637.

42 T. Ishida and S. Kato, J. Am. Chem. Soc., 2003, 125, 12035-12048.

43 A. V. Finkelstein, Protein physics; Academic press, Amsterdam, 2002.

${ }^{44}$ F. C. Bernstein, T. F. Koetzle, G. J. Williams, E. E. Meyer, M. D. Brice, J. R. Rodgers, O. Kennard, T. Shimanouchi, and M. Tasumi, J. Mol. Biol., 1977, 112, 535-542. 